\title{
Capacidad de agencia de autocuidado y calidad de vida en salud del paciente que acude al Servicio de Consulta Externa de Geriatría del Hospital Nacional Guillermo Almenara Irigoyen, Lima, 2015
}

\author{
Ability to self-care agency and health quality of life of patients attending the outpatient department of \\ geriatrics at Guillermo Almenara Irigoyen National Hospital, Lima, 2015
}

Fressia Angélica Olazo Obando', Maximina Contreras Castro²

\begin{abstract}
RESUMEN
Objetivo: El estudio tuvo como objetivo determinar la relación entre la capacidad de agencia de autocuidado y la calidad de vida en salud del paciente que acude al servicio de consulta externa de geriatría del Hospital Guillermo Almenara Irigoyen. Material y métodos: Fue de tipo cuantitativo, descriptivo y diseño correlacional; la muestra estuvo conformada por 175 pacientes con edades comprendidas entre 60 y 85 años, los datos fueron obtenidos a través de la escala ASA (Appraisal of Self-care Agency Scale) y el cuestionario SF-12 v2. Resultados: Los resultados indican que existe una relación directa y significativa entre la capacidad de agencia de autocuidado y la calidad de vida en salud (rho=0.284, $\mathrm{p}=0.000$ ), así como entre la capacidad de agencia de autocuidado y las dimensiones de vitalidad ( $r h o=0.430, p=0.000$ ), la salud general ( $r h o=0.249, p=0.001$ ), función física (rho=0.364, $\mathrm{p}=0.000)$ y la salud mental ( $\mathrm{rho}=0.250, \mathrm{p}=0.001)$; no se encontró relación significativa entre la capacidad de agencia de autocuidado y las dimensiones rol físico (rho=0.084, $\mathrm{p}=0.268$ ), rol emocional ( $\mathrm{rho}=0.101, \mathrm{p}=0.185$ ), dolor corporal (rho=0.092, $\mathrm{p}=0.225$ ) y funcionamiento social (rho=0.129, $\mathrm{p}=0.088$ ). Conclusiones: Se concluyó en que la capacidad de agencia de autocuidado y la calidad de vida en salud son variables que están relacionadas.
\end{abstract}

Palabras clave: Capacidad de agencia de autocuidado, calidad de vida en salud, adulto mayor.

\begin{abstract}
Objetive: The objective of the present study is to determine the relation between the capacity of self-care agency and life quality for the health of the elderly in the Outpatient Geriatric Service at the Guillermo Almenara Irigoyen Hospital. Methods: The study was descriptive, cross -sectional, with a correlation design in which 175 people participated, ages 60 to 85; data was obtained through the ASA Scale and the SF- 12 v2 Questionnaire. Results: The results indicate that there is a direct and significant relation between the capacity of self-care agency and the quality of life in health, (rho=0.284, $\mathrm{p}=0.000$ ), as well as in the capacity of self-care agency and the dimensions of vitality (rho $=0.430, p=0.000$ ), general health ( $r h o=0.249, p=0.001$ ), physical function ( $r h o=0.364, p=0.000$ ) and mental health $(\mathrm{rho}=0.250, \mathrm{p}=0.001)$; significant relation was not found between the agency capacity of selfcare and the dimensions of physical role ( $r h o=0.084, p=0.268$ ) emotional role (rho=0.101, $\mathrm{p}=0.185$ ), body pain (rho=0.092, $\mathrm{p}=0.225$ ) and social functioning ( $r h o=0.129, \mathrm{p}=0.088$ ). It is concluded that the capacity of self-care agency and the quality of life in health are related variables.
\end{abstract}

Keywords: Capacity of self-care agency, life quality in health, elderly

\footnotetext{
${ }^{1}$ Supervisora de Departamento de Enfermería, Hospital Nacional Guillermo Almenara Irigoyen, Lima, Perú.

${ }^{2}$ Doctora en Administración, Rectora de la Universidad Peruana Unión, Lima, Perú.
} 


\section{INTRODUCCIÓN}

El Perú experimenta un importante desarrollo económico que contribuye a un incremento de la esperanza de vida al nacer, al mejoramiento de la calidad de vida y mejores servicios, entre otros; sin embargo, paralelo a ello, persisten las brechas en sectores y poblaciones vulnerables, que generan diferencias y exclusión social; siendo uno de ellos el grupo constituido por los adultos mayores, con edades de 60 y más años (Instituto Nacional de Estadística e Informática [INEI], 2012).

Esta realidad genera la necesidad de conocer la calidad de vida en este grupo de personas, más allá del concepto y la forma de medirla (Gimeo, Pinazo, García, T. y García, A., 2008). Al considerar la percepción del paciente adulto mayor, sus deseos y motivaciones en el proceso de toma de decisiones en salud, hace que el tema tenga real importancia (Schwartzmann, 2003).

La preocupación sobre la salud considerada como algo más que la ausencia de la enfermedad, cobra vida en la actualidad como materia de prevención, debido a los factores determinantes presentados especialmente en la edad avanzada (Márquez y Garatachea, 2012).

En este contexto, el autocuidado parte del estilo de vida (MINSA, 2008), juega un rol importante en la prevención de los problemas ocasionados por los comportamientos que mantenga la persona. El servicio de Geriatría en Consulta Externa del Hospital Nacional Guillermo Almenara Irigoyen, atiende a pacientes adultos, quienes en su mayoría son portadores de patologías crónicas y necesitan adiestramiento o rehabilitación de las actividades perdidas por enfermedad, problemas funcionales de deterioro cognitivo; por ello la importancia de realizar el presente estudio.

\section{MATERIALES Y MÉTODOS}

El presente estudio fue de tipo descriptivo, diseño correlacional y de corte transversal. La población estuvo constituida por todos los pacientes geriátricos de 60 años a más, atendidos en la consulta externa del Hospital Nacional Guillermo Almenara Irigoyen, siendo un total de 320 .

La muestra estuvo conformada por 175 pacientes considerando criterios de inclusión y exclusión. El instrumento utilizado fue la escala de valoración de agencia de autocuidado Appraisal of Self-care Agency Scale (ASA), cuya confiabilidad fue de 9.91 según el Coeficiente alfa de Cronbach luego de una prueba piloto aplicada en 16 pacientes geriátricos de dicho nosocomio.

El instrumento para evaluar la calidad de vida fue un cuestionario (Iraurgi, 2007), conformado por 12 ítems con niveles de evaluación en escala de 1 a 5 para los ítems 1, 8 y 12; niveles de valoración en escala de 1 a 3 para los ítemes 2 y 3; valoración 1 y 2 para los ítemes 4, 5, 6 y 7; y valoración en escala de 1 a 6 para los ítems 9, 10 y 11. Los datos fueron procesados con el paquete estadístico SPSS, versión 22.0 .

\section{RESULTADOS}

Tabla 1

Capacidad de agencia de autocuidado de los adultos mayores que acuden a Consulta Externa de Geriatría del Hospital Nacional Guillermo Almenara Irigoyen, Lima 2015.

\begin{tabular}{lcc}
\hline \multicolumn{1}{c}{ Capacidad de Agencia de Autocuidado } & $\mathrm{n}$ & $\%$ \\
\hline Baja & 0 & 0.0 \\
Media & 159 & 90.9 \\
Alta & 16 & 9.1 \\
Total & 175 & 100.0 \\
\hline
\end{tabular}

La tabla 1 muestra que el $90.9 \%$ de los participantes presenta capacidad de agencia de autocuidado media y el 9.1\% capacidad de agencia de autocuidado alta. 
Capacidad de agencia de autocuidado y calidad de vida en salud del paciente que acude al Servicio de Consulta Externa de Geriatría del Hospital Nacional Guillermo Almenara Irigoyen, Lima, 2015

Tabla 2

Calidad de vida y sus respectivas dimensiones de los adultos mayores que acuden a Consulta Externa de Geriatría del Hospital Nacional Guillermo Almenara Irigoyen, Lima 2015.

\begin{tabular}{lccc}
\hline Calidad de vida & $\mathrm{n}$ & $\overline{\mathrm{x}}$ & $\mathrm{S}$ \\
\hline Salud general & 175 & 25.29 & 16.30 \\
Función física & 175 & 40.00 & 34.84 \\
Rol físico & 175 & 30.86 & 42.10 \\
Rol emocional & 175 & 41.43 & 45.62 \\
Dolor corporal & 175 & 57.71 & 24.66 \\
Salud mental & 175 & 55.83 & 18.48 \\
Vitalidad & 175 & 61.60 & 27.16 \\
Funcionamiento social & 175 & 52.43 & 20.70 \\
Calidad de vida & 175 & 44.44 & 22.52 \\
\hline
\end{tabular}

La tabla 2 muestra la media general con tendencia a una baja calidad de vida ( $\overline{\mathrm{X}}=44.44, \mathrm{~S}=22.52)$; $\sin$ embargo, se observa tendencia a una buena calidad de vida en las dimensiones dolor corporal $(\overline{\mathrm{X}}=57.71$, $\mathrm{S}=24.66)$, salud mental $(\overline{\mathrm{X}}=55.83, \mathrm{~S}=18.48)$, vitalidad $(\overline{\mathrm{X}}=61.60, \quad \mathrm{~S}=27.16) \quad \mathrm{y}$ funcionamiento social
$(\overline{\mathrm{X}}=52.43, \mathrm{~S}=20.70)$; tendencia a una baja calidad de vida en las dimensiones de salud general $(\overline{\mathrm{X}}=25.29$, $\mathrm{S}=16.30)$, función física $(\overline{\mathrm{X}}=40.00, \mathrm{~S}=34.84)$, rol físico $(\overline{\mathrm{X}}=30.86, \mathrm{~S}=42.10)$ y rol emocional $(\overline{\mathrm{X}}=41.43$, $\mathrm{S}=45.62)$.

Tabla 3

Relación entre capacidad de agencia de autocuidado y la calidad de vida de los adultos mayores que acuden a Consulta Externa de Geriatría del Hospital Nacional Guillermo Almenara Irigoyen, Lima 2015.

\begin{tabular}{lccc}
\hline \multirow{2}{*}{ Calidad de vida } & \multicolumn{3}{c}{$\begin{array}{c}\text { Capacidad de Agencia de } \\
\text { Autocuidado }\end{array}$} \\
\cline { 2 - 4 } & $\mathrm{n}$ & rho & $\mathrm{P}$ \\
\hline Salud general & 175 & 0.249 & 0.001 \\
Función física & 175 & 0.364 & 0.000 \\
Rol físico & 175 & 0.084 & 0.268 \\
Rol emocional & 175 & 0.101 & 0.185 \\
Dolor corporal & 175 & 0.092 & 0.225 \\
Salud Mental & 175 & 0.250 & 0.001 \\
Vitalidad & 175 & 0.430 & 0.000 \\
Funcionamiento social & 175 & 0.129 & 0.088 \\
Calidad de Vida & 175 & 0.284 & 0.000 \\
\hline
\end{tabular}

En la tabla 3 se observa una correlación directa y significativa para las variables capacidad de agencia de autocuidado y calidad de vida (rho $=0.284$, $\mathrm{p}=0.000$ ). En cuanto a la correlación de la capacidad de agencia de autocuidado y las dimensiones de la calidad de vida, se observa una correlación directa y significativa con la dimensión vitalidad $(\mathrm{rho}=0.430$, $\mathrm{p}=0.000)$, salud general $(\mathrm{rho}=0.249, \mathrm{p}=0.001)$, función física (rho $=0.364, \mathrm{p}=0.000$ ) y salud mental (rho=0.250, $\mathrm{p}=0.001)$; sin embargo, no se encontró relación significativa con las dimensiones rol físico (rho $=0.084, \mathrm{p}=0.268)$, rol emocional (rho $=0.101$, $\mathrm{p}=0.185)$, dolor corporal (rho $=0.092, \mathrm{p}=0.225)$ y funcionamiento social (rho $=0.129, \mathrm{p}=0.088$ ). 


\section{DISCUSIÓN}

En el estudio, el $90.9 \%$ de los pacientes adultos mayores presentó una capacidad de agencia de autocuidado media y sólo un 9.1\%, una capacidad de autocuidado alta, resaltando que ninguno presentó autocuidado bajo. El porcentaje obtenido para el nivel alto de la capacidad de agencia de autocuidado es muy bajo al ser comparado con los resultados encontrados por Cely, Espinosa \% Mateus (2008) y Rebolledo (2010) quienes incluyeron a personas de 18 a 28 años; lo que puede ser explicado por las características de las muestras, debido a que los jóvenes tienen todas sus capacidades en el nivel máximo de desarrollo, a diferencia de los adultos mayores que participaron en el presente estudio quienes experimentan una disminución de sus facultades y capacidades con el pasar de los años sustentadas por las teorías del envejecimiento (Blazer \& Steffens, 2010).

Asimismo, Burke (1998) sostiene que los cambios que se producen en el proceso del envejecimiento y que generalmente van acompañados de desaliento y depresión por la falta de logros, contribuyen al aumento de la incapacidad para realizar actividades propias de su cuidado. Sin embargo, es importante resaltar el porcentaje alto en el nivel medio de autocuidado encontrado en los adultos mayores, lo que refleja el sentido de responsabilidad desarrollado para cuidar su salud a pesar del cambio de conductas y actitudes que acompañan al envejecimiento (Rowe \& Kahn, 1998).

En cuanto a las puntuaciones encontradas con tendencia a una buena calidad de vida en las dimensiones de dolor corporal, salud mental, vitalidad y función social, Comming (1961) manifiesta que es propio del adulto mayor haber aprendido a manejar las situaciones que alteran su bienestar, tomar sus propias decisiones, optimizar la vitalidad que posee, al mismo tiempo que va cediendo el espacio social a la gente más joven; además, desarrolla actividades compatibles con su vitalidad y trata de seguir siendo miembro productivo de la sociedad hasta muy entrados el octavo y noveno decenios de vida.

Por otro lado, es preocupante encontrar que la dimensión salud general en el presente estudio y los estudios revisados, presentan las puntuaciones más bajas en todos los grupos etarios, esto puede ser explicado por las interpretaciones que da cada persona a la vida, que muchas veces son afectadas por la cultura y el entorno donde se desarrolla. Por tanto, es importante trabajar en la mejora de la dimensión salud general y reforzar las capacidades para percibir su salud como una realidad objetiva.
La relación significativa encontrada entre la capacidad de agencia de autocuidado y la calidad de vida en salud en los pacientes adultos mayores, indica que la compleja capacidad desarrollada por este grupo etario para discernir los factores que deben ser controlados o tratados para regular su propio funcionamiento y desarrollo (Orem, 1993), juega un papel preponderante en la forma como se enfrenta a la enfermedad, expresado en la evolución del estado de salud y la estrategia empleada no solamente para prolongar la vida, sino también para aliviar los síntomas y mantener el funcionamiento vital (Alfonso, Feísto, Llopiz \& Hernández, 2012).

La relación directa positiva y significativa encontrada entre la capacidad de agencia de autocuidado y la calidad de vida en las dimensiones de salud general, función física, salud mental y vitalidad, indica que a mayor capacidad de agencia de autocuidado, el adulto mayor va a percibir una mejor calidad de vida en estas dimensiones; por el contrario, a menor capacidad de agencia de autocuidado, va a percibir una menor calidad de vida en salud especialmente en la evaluación subjetiva que hace del estado de su salud global, que involucra las reacciones frente al dolor, percepción de dificultades, nivel de esfuerzo y nivel de preocupación por el estado de salud; así como, en la evaluación de los componentes emocionales de bienestar psicológico y en el sentimiento de energía y vitalidad frente al sentimiento de cansancio y agotamiento (Ruiz \& Pardo, 2005).

Es importante considerar que el déficit de autocuidado se traduce en enfermedades que causan deterioro de la salud y muerte (Franco, 1993). Los daños causados por la enfermedad, a la larga se convierten en limitantes para que la persona pueda ejercer su autocuidado de manera eficiente en la edad avanzada. Las limitaciones del movimiento que padece una persona, llamada también deterioro de la movilidad física, trae como consecuencia déficit de autocuidado (Lacida, 2007).

Considerando estas evidencias y el incremento anual de la población de 60 años a más en el mundo y que el Perú no es ajeno a este fenómeno social según el Ministerio de la Mujer y Poblaciones Vulnerables (MIMP, 2014) y teniendo en cuenta que muchas veces las causas de enfermedad o muerte que podrían ser evitables, están relacionadas con el estilo de vida (OPS, 2014), es importante que el personal de salud asuma su responsabilidad fomentando el autocuidado a través de la educación y el testimonio de vida sana (Tobón, 2003), que llevará a los adultos mayores a disfrutar esta etapa de su vida con calidad (Viguera, 2015). 
Este reto requiere de cuidado multidisciplinario efectivo, que incluya no sólo la recuperación sino también la incorporación destacada de la promoción de la salud, el crecimiento individual y familiar (Marín, 2000), que debe ser atendido a través de programas orientados a fortalecer las habilidades para ejercer el propio cuidado.

\section{Declaración de financiamiento y de conflicto de intereses:}

El estudio fue financiado por los autores, quienes declaran no tener algún tipo de conflicto de interés en la investigación realizada.

\section{Correspondencia:}

Fresia Olazo Obando

Hospital Nacional Guillermo Almenara Irigoyen. Av. Grau Nº 800, Lima, Perú

e-mail: fressiaolaz@hotmail.com

\section{REFERENCIAS BIBLIOGRÁFICAS}

Alfonso, A., Feísto, T., Llopiz, L. \& Hernández, P. (2012). Calidad de vida en pacientes con isquemia crónica de miembros inferiores tratados con células madre adultas. Revista Cubana de Hematología. 28(4), 398403. Recuperado de http://www.medigraphic.com/ pdfs/revcubheminmhem/rch-2012/rch124h.pdf

Burke, M. (1998). Enfermería gerontológica: cuidados integrales del adulto mayor. España: Elsevier.

Comming, E. (1961). Growing old: The process of desenagement. Basic Books, New York.

Cely, M., Espinosa, X. \& Mateus, J. (2008). Capacidad de agencia de autocuidado de las personas portadoras de VIH/SIDA en una fundación de Bogotá, en el periodo comprendido entre agosto a noviembre de 2007 (Tesis de licenciatura). Recuperado de http://www.javeriana. edu.co/biblos/tesis/enfermeria/tesis12.pdf

Franco, E. (1993). Indicadores de atención primaria en salud. Recuperado de http://biblioteca.usac.edu.gt/ tesis/05/05_6683.pdf

Gimeo, M., Pinazo, D., García, T. \& García, A. (2008). Evaluación de las organizaciones saludables. Una propuesta metodológica. España: Diseñarte-Goaprint. Recuperado http://www.orpconference.org/2008/ actividades/documentar/organizacionsaludable

Instituto Nacional de Estadística e Informática. (2012). Perú: Demanda de atención del adulto mayor por problemas de salud 2006-2010. Perú: INEI. Recuperado http://www.inei.gob.pe/media/MenuRecursivo/ publicaciones_digitales/Est/Lib1041/libro.

Lacida, M. (2007). Deterioro de la movilidad física y continuidad de cuidados. Biblioteca Las Casas. Recuperado: http://www.index-f.com/lascasas/ documentos/lc0235.

Iraurgi, I. (2007). Comparación del instrumento de salud
SF-12 frente al SF-36 en pacientes en mantenimiento con metadona. Adicciones. 9(1), 59-67. Recuperado de http://www.adicciones.es/files/Iraurgi.pdf

Marín, P. (2000). Manual de geriatría y gerontología. Recuperado de http://escuela.med.puc.cl/publ/ manualgeriatria/pdf/cuidadosenfermeria.pdf

Márquez, S. \& Garatachea, N. (2012). Actividad física y Salud. España: Ediciones Díaz Santos.

MINSA. (2008). Yo cuido mi salud: Consejos saludables del cuidado y autocuidado para el adulto mayor. Dirección de atención integral de salud. Recuperado de http://www.minsa.gob.pe/portada/.../ROTAFOLIO_ AUTOCUIDADO.doc.

MIMP. (2014). Acción internacional de la mujer y la población. Cuaderno sobre poblaciones vulnerables. Recuperado de http:/www.mimp.gob.pe/files/mimp/ especializados/boletines_dvmpv/cuaderno-14-dvmpv. pdf

OPS. (2014). Autocuidado: Clave para una buena calidad de vida en los adultos mayores. Recuperado de http:// www.paho.org/arg/index.php?option=com_content \&view=article\&id=1289:autocuidado-clave-buenacalidad-vida-adultos-mayores\&Itemid=290

Orem, D. (1993). Modelo de Orem: Conceptos de enfermería en la práctica. España: Editorial Masson S. A.

Rebolledo, D. (2010) Autocuidado en los estudiantes de la Carrera de enfermería de la Universidad del Bío Bío: un análisis según el modelo de autocuidado de Dorotea Orem (Tesis de licenciatura). Recuperado de http://cybertesis.uach.cl/tesis/uach/2010/fmr292a/doc/ fmr292a.pdf

Rowe, JW \& Kahn, RL. (1998). Successful aging. Dell Publishers, New York. 
Ruiz, M. \& Pardo, A (2005). Calidad de vida relacionada con la salud: definición y utilización en la práctica médica. PharmacoEconomics Spanish Research Articles. 2(1), 31-43. Recuperado de http://link. springer.com/article/10.1007/BF03320897

Schwartzmann, L. (2003). Calidad de vida relacionada con la salud: aspectos conceptuales. Ciencia y Enfermería. 9(2), 9-21. Recuperado de http://www. psicologiamedica.org.uy/pdfs/cvrs.pdf.
Tobón, O. (2003). El autocuidado una habilidad para vivir. Revista Hacia la Promoción de la Salud [serie en línea]. Recuperado de http://promocionsalud.ucaldas. edu.co/downloads/Revista\%208_5.pdf

Viguera, V. (2015). El estrés y el autocuidado: trabajando con adultos mayores. Tiempo el portal de la Psicogerontología. 32. Recuperado de http://www. psicomundo.com/tiempo/tiempo32/viguera.htm.

Recibido: 25/08/2015 Aceptado: 20/11/2015 